\title{
Evaluation of Diabetic Nephropathy using Endogenous Plant Portulaca grandiflora Extract in Alloxan Induced Diabetic Rats
}

\author{
Ch. Pradeep Kumar, G. Sandhya Rani* \\ Department of Pharmacology, Vaageswari College of Pharmacy, Karimnagr - 505 001, Telangana, India.
}

\section{ART ICLE DETAILS}

\section{Article history:}

Received 23 September 2019

Accepted 18 October 2019

Available online 21 October 2019

\section{Keywords:}

Diabetic Nephropathy

Alloxan Monohydrate

Portulaca grandiflora

\begin{abstract}
A B S T R A C T
The aim of the study is to prevent nephropathy by using endogenous plant extract Portulaca grandiflora in alloxan induced diabetic rats. Albino rats weighing 150-200 g were divided into 4 groups, each group consists of 4 rats. The alloxan monohydrate (ip. $150 \mathrm{mg} / \mathrm{kg}$ ) was used for induction of diabetes. After 2 days, fasting blood sugar was assessed and rats with FBS $>250 \mathrm{mg} / \mathrm{dL}$ were considered as diabetic. After 1 week of alloxan injection the blood urea, creatinine, glucose and uric acid were determined for confirmation of nephropathy. Then these animals were treated with Portulaca grandiflora plant extract. The glimepiride $(2 \mathrm{mg} / \mathrm{kg})$ was used as standard drug. After a single dose of alloxan monohydrate blood samples were collected on initial and 7th day from all the rats and levels of glucose, urea, uric acid and creatinine were examined. Then animals were scarified and kidney tissue of control, inducing test, standard groups are examined by histopathology. The results given away that oral administration of Portulaca grandiflora plant extract $(100 \mathrm{mg} / \mathrm{kg}$ and $200 \mathrm{mg} / \mathrm{kg}$ ) was significantly reduces the levels of glucose, uric acid, creatinine, urea in diabetic rats. This study revealed that the Portulaca grandiflora is useful in treating diabetes and having minimizing activity of complication diabetic nephropathy by its anti-oxidant activity. Further research is required for better results for diabetic complications.
\end{abstract}

\section{Introduction}

Diabetes is a metabolic disorder which is not fatal but complications arising from this disease conditions are fatal. So, treating complications can decrease the risk of death in diabetic patients. Literature survey revealed that Portulaca grandiflora is a traditionally used medicinal plant. The ethnobotanical survey reveals that in India the plant is used in the treatment of cirrhosis of the liver, pharyngeal pain and swelling, leaves used for scurvy and juice of leaves and stems are used for scalds, burns eczema, also having the property depurative and antioxidant activity [1-4]

Portulaca grandiflora belongs to the family Portulacaeae and it is commonly known as Portulaca, moss rose. It is a low, fleshy, trailing perennial herb, attaining a height of 15-30 centimeters. Stems are slender or ascending, with hairy joints. Leaves are alternate, small, fleshy, clustered and 2 centimeters long. Flowers are showy, terminal, up to 3 centimeters across, subtended by clustered leaves. Petals are five red, white, yellow, orange, pink opening in mid-morning and closing by midafternoon $[5,6]$.

Portulaca grandiflora is also used in the treatment of cancer through nanotechnology [7]. Medicinal plants are the main source of organic compounds such as polyphenolic acid, triterpenoids, carotenoids, polysaccharides, reducing agents, sterols and flavonoids etc., [8-10]. These organic compounds represent a source for the discovery and development of new types of antidiabetic molecules. Many compounds isolated from the plant sources have been reported to show anti diabetic activity.

The present investigation was undertaken with a view to provide scientific evidence for its traditional use in the treatment of diabetic nephropathy. It deals the effect of Portulaca grandiflora extract on diabetic nephropathy.

\section{Experimental Methods}

\subsection{Collection of Plant Material}

The leaves of Portulaca grandiflora plant were collected from the village Mangalpelly Mandal, Choppadandi, Karimnagar district, Telangana, India.
It was authenticated by botanical survey of India, Deccan regional Centre, Attapur, Hyderabad (Authentication no: BSI/DRC/2018/-19/Tech./795).

\subsection{Plant Processing}

The leaves of Portulaca grandiflora were shade dried and powdered by mechanical grinder. Powder is passed through the sieve no: 30. Further extraction procedure is done by the solvent ethanol using Soxhlet apparatus.

\subsection{Soxhlet Extraction of Plant Material}

In this method, the finely grounded crude drug was placed in a porous bag or thimble, placed in chamber $\mathrm{E}$ of the Soxhlet apparatus. The extracting solvent heated in the flask A and vapours condensed in condenser D. The condensed extractant is drained by a small drop of liquid into the thimble containing the crude drug. The level of liquid in chamber E rises to the top siphon tube $\mathrm{C}$, the liquid contents of chamber $\mathrm{E}$ siphon into flask A. this process is continued until the last drop of the solvent from the siphon tube does not leave the residue. The benefit of this method is bulk amount of the drug is extracted with the smaller quantity of the solvent. The extracts obtained are kept in desiccator to free from contamination and further extract is used for experimental procedure.

\subsection{Phytochemical Screening}

The ethanol extract of the samples was analyzed to asses for the presence of secondary metabolites such as flavonoid, polyphenols, tannins, alkaloids, carbohydrates, amino acids, volatile oils and saponin glycosides by the standard methods [11].

\subsection{Animals}

Albino rats (Wister) weighing 150-200 g are used for the experiment. The animals are maintained under standard temperature $26-28{ }^{\circ} \mathrm{C}$ and humidity $\left(35-60^{\circ} \mathrm{C}\right)$ throughout the period of experimental study. Animals were provided standard rodent pellet diet and water.

\subsection{Acute Toxicity Study}

The Wister rats of both sexes was divided into five groups containing each 12 animals. Group 1-water control $5 \mathrm{~mL} / \mathrm{kg}$ per day. Group 2, 3, 4, 5- 
Animals received orally water suspension of Portulaca grandiflora extracts of doses $10,100,2000 \mathrm{mg} / \mathrm{kg}$ respectively [1].

Body weights, food intake, signs of animals were observed daily basis, through the study. At the end of $7^{\text {th }}$ day, 1-4 groups of rats were fasted from $18 \mathrm{~h}$, then anesthetized with ether and scarified, by withdrawing the blood and biochemical parameters was measured. By the consumption the Portulaca grandiflora we don't find any remarkable toxic effects.

\subsection{Method for Inducing Diabetes and Complications}

Diabetic complications were induced in Wister rats by administration of alloxan monohydrate dose of $150 \mathrm{mg} / \mathrm{kg}$ intraperitoneally in normal saline. After one hour of alloxan administration the animals were given feed ad libitum. The animals were kept fasting overnight and blood glucose levels are estimated before and after 72 hours of alloxan treatment. Animals showing blood glucose levels of $>200 \mathrm{mg} / \mathrm{dL}$ is considered as diabetic and used for the study for 7 days. Diabetic rats were divided in to four groups with each group four animals.

Group 1: Rats served as normal control group.

Group 2: Rats served as diabetic disease control.

Group 3: Diabetic rats treated with Portulaca grandiflora plant extract at a dose of low $-100 \mathrm{mg} / \mathrm{kg}$ and high $-200 \mathrm{mg} / \mathrm{kg}$

Group 4: Diabetic rats treated with glimepiride standard drug at 2 $\mathrm{mg} / \mathrm{kg}$.

\section{Results and Discussion}

\subsection{Phytochemical Screening}

The phytochemical screening of Portulaca grandiflora leaf extract revealed the presence of flavanoids, tannins, polyphenols, terpenoids alkaloids, amino acids and volatile oils (Table 1 ).

Table 1 Phytochemicals presece in Portulaca grandiflora

\begin{tabular}{ll}
\hline Constituent & Result \\
\hline Flavanoid & + \\
polyphenols & + \\
Tannins & + \\
Alkaloids & + \\
carbohydrates & + \\
Amino acids & + \\
Volatile oils & + \\
Saponin Glycosides & - \\
\hline
\end{tabular}

\subsection{Acute Toxicity}

No animals were died during the toxicity studies are administered with Portulaca grandiflora at the dose of $2000 \mathrm{mg} / \mathrm{kg}$.

\subsection{Effect on Serum Glucose, Creatinine, Uric Acid and Urea Levels}

Effect of Portulaca grandiflora on serum glucose levels $(\mathrm{mg} / \mathrm{dL})$ in diabetic rats has been studied. By inducing alloxan monohydrate, the glucose levels are elevated than the normal range in every group. The plant Portulaca grandiflora (100 mg/kg and $200 \mathrm{mg} / \mathrm{kg}$ ) was administered to the group 3 and standard drug glimipiride ( $2 \mathrm{mg} / \mathrm{kg}$ ) was administered to group 4. From Table 2, upon treating with plant extract Portulaca grandiflora and standard drug the glucose levels were decreased significantly and creatinine levels were reduced considerably. The plant extract and standard drug decreases the uric acid and urea levels significantly.

Table 2 Effect of Portulaca grandiflora on serum parameters (mg\%)

\begin{tabular}{lllll}
\hline Groups/Interval & Glucose & \multicolumn{3}{l}{ Creatinine } \\
\cline { 2 - 5 } & 0-day & 7 -day & 0-day & 7-day \\
\hline Control & $78.3 \pm 3.62$ & $72.5 \pm 3.22$ & $1.63 \pm 0.15$ & $1.53 \pm 0.15$ \\
Inducing & $70.5 \pm 4.11$ & $176.7 \pm 5.96$ & $1.82 \pm 0.31$ & $2.03 \pm 0.20$ \\
Standard & $85.7 \pm 6.03$ & $150.3 \pm 5.47$ & $1.66 \pm 0.14$ & $1.70 \pm 0.20$ \\
Test & $85 \pm 4.49$ & $163.6 \pm 8.1$ & $1.73 \pm 0.21$ & $1.80 \pm 0.37$ \\
\hline & Uric acid & & Urea & \\
Control & $2.7 \pm 0.19$ & $2.9 \pm 0.07$ & $10.9 \pm 0.51$ & $10.6 \pm 0.57$ \\
Inducing & $3.1 \pm 0.55$ & $4.9 \pm 0.46$ & $12.4 \pm 1.6$ & $20.3 \pm 4.18$ \\
Standard & $3.0 \pm 0.21$ & $3.15 \pm 0.20$ & $11.8 \pm 0.8$ & $12.4 \pm 1.02$ \\
Test & $2.8 \pm 0.41$ & $3.7 \pm 0.25$ & $11.7 \pm 1.5$ & $13.9 \pm 0.45$ \\
\hline All & &
\end{tabular}

All values of mean $\pm \operatorname{SEM}(n=4) *$ indicates $p<0.01 * *$ indicates $p<0.001$ vs. diabetic rat.

\subsection{Kidney Histopathology Studies}

After the histopathological examination the kidney from control A have adequate number of glomeruli and the bowmen's space was in normal https://doi.org/10.30799/jnpr.082.19050204 limits (Fig. 1). But the kidneys from the induced group B has marked alteration where the glomeruli and the bowmen's space in increased. Tubular epithelium is also degenerated.
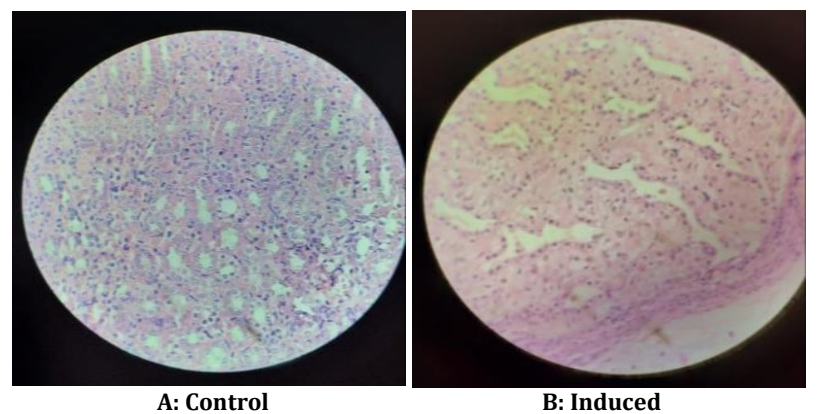

B: Induced

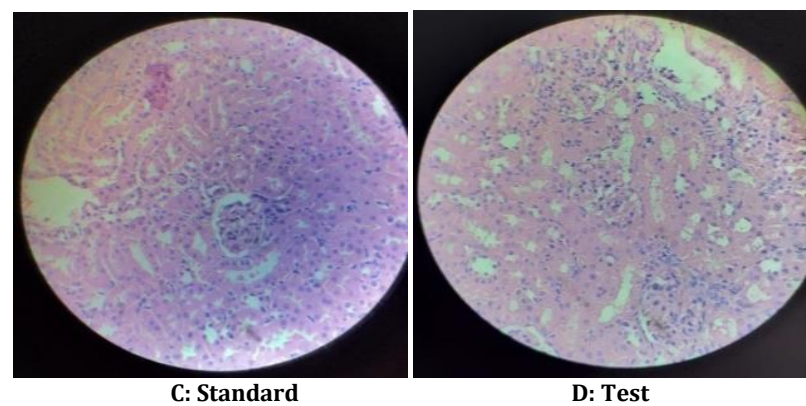

Fig. 1 Histopathological examination images

Kidneys which are from test group D have similar morphology to that of normal morphology. But tubular degeneration is observed. This indicates some protection activity is observed against diabetes induced kidney disease. Kidneys from standard group C showed normal architecture as similar as control.

The present results were meant to assess the anti-diabetic activity and its complications like diabetic nephropathy by using a plant extract Portulaca grandiflora, as the plant is having many medicinal values such as antioxidant activity, antibacterial activity, disinfectant etc. This diabetic and nephropathy activity is precise by estimating biomarkers be found of blood glucose levels, uric acid, creatinine, urea as well as histopathological examination in experimental rats.

\section{Conclusion}

Portulaca grandiflora plant extract has many medicinal properties to cure scurvy, scalds, burns, muscle spasms, can also treat cancer. The main chemical constituent of plant is betaxanthin having property to reduce cholesterol levels thereby preventing the atherosclerosis. In the current study alloxan induced rat model was used. Alloxan to produce increased blood glucose amount that generates diabetes, which in turn causes rigorous injure to the kidneys. The consequences promote increase in urea, and creatinine levels in blood. Treatment with Portulaca grandiflora extract decreases the levels of creatinine and urea, uric acid by its antioxidant activity

The histopathology reports of kidneys treated with alloxan monohydrate $(150 \mathrm{mg} / \mathrm{kg}$ ip.) enhanced blood glucose levels caused by diabetes and its complications are reported. The treatment is specified by the means of the plant extract Portulaca grandiflora and standard drug glimepiride ( $2 \mathrm{mg} / \mathrm{kg}$ ), so the plant extract is having defending effect on diabetes induced nephropathy same that of glimepiride.

\section{References}

[1] P. Chavalittumrong, S. Chivapat, A. Attawish, J. Bansiddh, S. Phadungpat, et al., Chronic toxicity study of Portulaca grandiflora Hook, Ethnopharmacol. 90(2004) 375-380

[2] D. Liu, X. Yin, H. Wang, Y. Zhou, Y. Zhang, Antimutagenicity screening of water extracts from 102 kinds of Chinese medicinal herbs, Zhongguo Zhong Yao Za Zhi 15 (1990) 617-622.

[3] M. Valko, D. Leibfritz, J. Moncol, T.D.M. Cronin, M. Mazur, J. Telser, Free radicals and antioxidants in normal physiological functions and human disease, Int. J. Biochem. Cell Biol. 39 (2007) 44-84.

[4] Y.Z. Cai, Q. Luo, M. Sun, H. Corke, Antioxidant activity and phenolic compounds of 112 traditional Chinese medicinal plants associated with anticancer, Life Sci. 74 (2004) 2157-2184.

[5] USDA, Portulaca grandiflora - Natural resources conservation service, Plants Database, USDA Plant Hardiness Zone Map, USA, 2016. 
[6] S.B. Patil, A.D. Jagdale, D.B. Patil, A.S. Kulkarni, S.P. Govindwar, Evaluation of fluoride uptake and accumulation by Portulaca grandiflora Hook. and Brassica oleracea Linn. from water, Int. J. Sci. Eng. Tech. Res. 3(12) (2014) 3474-3477.

[7] M. Valko, C.J. Rhodes, J. Moncola, M. Izakovic, M. Mazura, Mini-review: Free radicals, metals and antioxidants in oxidative stress-induced cancer, Chem-Biol Interact. 160 (2006) 1-40.

[8] Choon Kiat Lim, Wen Ni Tiong, Joo Ling Loo, Antioxidant activity and total phenolic content of different varieties of Portulaca grandiflora, Int. J. Phytopharm. 4(1) (2017) 01-05.
[9] P. Chavalittumrong, B. Sriwanthana, A. Rojanawiwat, R. Kijphati, B. Jitjuk, et al., Safety of the aqueous extract of Portulaca grandiflora Hook in healthy volunteers, Songklanakarin J. Sci. Technol. 29 (2007) 95-100

[10] Ayumi Ohsaki, Yukihiro Asaka, Takashi Kubota, Kozo Shibata, Takashi Tokoroyama, Portulene acetal, a novel minor constituent of Portulaca grandiflora with Significance for the biosynthesis of portulal, J. Nat. Prod. 60(9), (1997) 912-914

[11] Indian Pharmacopoeia, Vol. 2, 3rd Ed., Ministry of Health, Controller of publications, Govt. of India, New Delhi, 1985, pp.A74 -A75. 